\title{
Ketamine Infusions for Refractory Complex Regional Pain Syndrome: A Review
}

\author{
Jordon Lui, B.Kin ${ }^{1}$ \\ ${ }^{1}$ Faculty of Medicine, University of British Columnia
}

A BSTRACT

Complex regional pain syndrome (CRPS) is a debilitating pain disorder that is often resistant to conventional treatment options. Although the precise pathophysiology of CRPS has not yet been fully elucidated, it is thought that central sensitization through the proliferation of $\mathrm{N}$-methyl-D-aspartate (NMDA) receptors leads to the amplification of pain transmission within the central nervous system. Accordingly, the NMDA antagonist ketamine has been employed for its potential ability to reverse central sensitization and provide analgesia. Promising studies have shown that ketamine, when administered through prolonged intravenous infusions, may be effective for relieving pain in cases of refractory CRPS. Currently, ketamine infusions for CRPS are offered at select pain clinics in North America. However, it should be emphasized that evidence from high-quality trials is lacking and unresolved concerns over potenial neurotoxicity, urological toxicity, and hepatotoxicity of prolonged ketamine use remain. These concerns render the long-term use of ketamine questionable. Therefore, ketamine infusions should be used with caution, and may be a reasonable therapeutic option only for refractory cases of CRPS. Proper monitoring for signs of toxicity must be ensured. In addition, a physical intervention program should be used in conjunction with ketamine to fully restore function and quality of life for refractory CRPS patients.

\section{RÉ S U MÉ}

Le syndrome douloureux régional complexe (SDRC) est un trouble de douleur débilitante qui est souvent résistant à des options de traitements conventionnels. Malgré le fait que la pathophysiologie précise du SDRC n'a pas encore été confirmée, certains chercheurs pensent qu'il y a une sensibilisation centrale par l'intermédiaire de la prolifération de récepteurs N-méthyl-D-aspartate (NMDA) qui conduisent à l'amplification de la transmission de la douleur dans le système nerveux central. En conséquence, l'antagoniste-NMDA, la kétamine, a été utilisé pour sa capacité de renverser la sensibilité centrale en créant un effet analgésique. Des études prometteuses ont démontré que la kétamine, lorsqu'administrée par des infusions intraveineuses prolongées, pourrait s'avérer efficace pour apaiser la douleur dans des cas de SDRC réfractaires. Des infusions de kétamine pour le SDRC sont offertes actuellement à des cliniques de douleur spécifiques en Amérique du Nord. Toutefois, il manque d'essais randomisés de qualité pour démontrer clairement l'efficacité de ce traitement. Des connaissances plus approfondies sur le potentiel neurotoxique, la toxicité urologique, et l'hépatotoxicité de l'utilisation prolongée de kétamine sont nécessaires. Ainsi, les infusions de kétamine doivent être utilisées avec précaution et ont un potentiel thérapeutique seulement pour les cas réfractaires de SDRC. Il est important d'assurer un suivi adéquat pour des signes de toxicité. De plus, un programme d'intervention physique devrait également être offert en plus des traitements de kétamine pour optimiser la fonction et la qualité de vie des patients avec un SDRC réfractaire.

\section{INTRODUCTION}

Complex regional pain syndrome (CRPS) is a severe pain disorder that is often characterized by chronicity and significant functional impairments [1]. First described over 160 years ago, the condition was assigned a variety of terms including: "neurovascular dystrophy", "algodystrophy", "causalgia", and "reflex sympathetic dystrophy" [2]. In 1994, the International Association for the Study of Pain (IASP) unified these terms by introducing "CRPS" as a new diagnostic label. The existence of such disparate termi- nology in the past reflects our evolving, yet incomplete, understanding of this disorder. To this day, CRPS is difficult to treat, and remains a significant barrier to rehabilitation.

The IASP currently defines CRPS as "an array of painful conditions that are characterized by a continuing (spontaneous and/or evoked) regional pain that is seemingly disproportionate in time or degree to the usual course of any known trauma or other lesion" [3]. CRPS most commonly occurs after traumatic events-in fact, up to $46 \%$ of cases can be attributed to bone fractures or

Keywords: Complex regional pain syndrome; Ketamine; Chronic pain 
Table 1. Efficacy of current therapies for CRPS: abstracted from recent systematic reviews [10-12].

\begin{tabular}{|c|c|c|}
\hline \multicolumn{2}{|c|}{ Evidence for Efficacy in CRPS } & \multirow{2}{*}{$\begin{array}{c}\text { Effective in patients with evidence of } \\
\text { Drugs }\end{array}$} \\
\hline bisphos demineralization
\end{tabular}

Abbreviations: IV, intravenous; N/A, not available; PO, oral; TCA, tricyclic antidepressants; SSNRI, selective serotonin-norepinephrine reuptake inhibitor; IVRSB, intravenous regional sympathetic block.

crush injuries [4]. In addition, it may develop secondary to spinal cord injury, peripheral neuropathies, prolonged immobilization, brain injury, and even vascular events such as stroke and myocardial infarction $[5,6]$. The incidence and prevalence of CRPS in Canada is currently unknown. However, current estimates suggest that the overall incidence in the U.S. is at least 50,000 cases per year [2]. It has also been suggested that the true incidence may be even higher due to the under-recognition of this diagnostically challenging condition [7].

Clinically, CRPS usually presents in the extremities with a wide spectrum of signs and symptoms. Common characteristics can include allodynia, hyperalgesia, motor dysfunction, autonomic abnormalities (e.g., swelling, sweating, skin blood flow changes), or trophic changes such as muscle wasting and bone demineralization [5]. Further investigations may or may not reveal an identifiable nerve lesion. The exact pathophysiological mechanisms underlying these changes in CRPS are unknown but research in the last few decades suggests a multifactorial process [8,9]. Briefly, the development of CRPS may involve sympathetic nervous system dysfunction, neurogenic inflammation, autoimmune factors, psychological factors, and peripheral and central sensitization [8,9]. Unfortunately, due to the difficulty in identifying the underlying mechanisms, this condition is difficult to treat. Current therapeutic options that target these underlying mechanisms are limited and often fail to provide pain relief. In addi- tion, evidence for conventionally used CRPS treatments, such as sympathetic nerve blockade, anticonvulsants, antidepressants, and opioids, has been largely insubstantial (Table 1) [10-12]. This means that a significant portion of CRPS patients can remain refractory to conventional treatments and may suffer long-term pain and disability.

There is a clear need for developing and evaluating novel treatment options for CRPS. Indeed, recent work has focused on therapy targeted against the role of central sensitization in CRPS. Although the pathophysiology is not fully understood, there is strong evidence that central sensitization is a key mechanism involved in both the induction and maintenance of pain in CRPS [13]. Central sensitization is a process which leads to enhanced transmission of excitatory signals in afferent pain pathways and therefore amplification of painful stimuli [14]. This process is thought to be mediated by the overactivation of N-methyl-Daspartate (NMDA) receptors following prolonged tissue insult. Accordingly, the NMDA receptor antagonist ketamine has been employed for its potential ability to block these receptors, thus reversing central sensitization and ultimately providing pain relief [14].

A growing body of evidence suggests that ketamine, when administered via an intravenous (IV) infusion, may be effective in treating refractory CRPS [15-18]. However, current guidelines do 
not recommend the use of ketamine in the routine management of CRPS due to a lack of high-quality evidence and concerns over long-term safety [19]. Despite these concerns, the use of IV ketamine is becoming increasingly accepted among pain specialists and is often employed off-label in select North American clinics, especially the U.S. It is therefore essential that physicians and patients are aware of the current evidence on the safety and efficacy of ketamine infusions for CRPS.

\section{METHODS}

A narrative review was conducted by searching English-language articles in PubMed until January 2015. The main search terms were: complex regional pain syndrome, ketamine, infusion, chronic pain, refractory, central sensitization, and safety. To examine the efficacy of ketamine infusions, only randomized controlled trials were selected for further discussion. However, one prospective trial was also included for illustrative purposes. To examine the long-term safety of ketamine, all article types including case series and cohort studies were selected.

\section{KETAMINE}

Ketamine is an anesthetic agent that was first introduced to clinical practice in the 1960s for its effective analgesic and sedative effects [20]. Since then, it has also been associated with shortterm psychotomimetic side effects including: hallucinations, psychosis, euphoria, anxiety, and agitation. In fact, it was once administered to experimentally model schizophrenia [20]. Unfortunately, the side effects of ketamine have also made it a popular drug of abuse, mainly for its hallucinogenic and euphoric properties. A recent report ranked ketamine as the fourth most widely used club drug in the United Kingdom, after cannabis, ecstasy, and cocaine [21].

Pharmacologically, ketamine is complex as it interacts with a variety of receptors including opioidergic, muscarinic, and monaminergic receptors [14]. However, the analgesic efficacy of ketamine is presumably due to its non-competitive antagonism of NMDA receptors in the central nervous system [14]. In response to tissue or nerve damage, repeated nociceptive input may lead to the release of neuromodulators such as substance $P$, bradykinin, prostaglandins, and glutamate. This prolonged excitatory input results in the withdrawal of normal tonic inhibition of NMDA receptors, facilitating long-term potentiation of the neural circuitry responsible for pain and contributing to the allodynia and hyperalgesia typically observed in CRPS [14]. Ketamine is the most potent NMDA receptor antagonist available and it is purported to counter central sensitization by inhibiting the latter mechanism [14].

In the setting of chronic pain management, various forms of ket- amine administration have been investigated. Oral bioavailability is poor and topical formulations are relatively understudied $[22,23]$. Prolonged IV infusions, typically spanning multiple days, have shown the most promising efficacy. In the management of acute pain, it has been observed that the analgesic effects of ketamine can often persist beyond what is expected for the halflife of the drug [24]. Therefore, it is speculated that prolonged IV infusions may effectively promote long-term desensitization of NMDA receptors, leading to analgesic effects that may outlast the duration of the infusions [14].

\section{EVIDENCE FOR KETAMINE EFFICACY}

Numerous studies have investigated the use of ketamine infusions in CRPS [15-17]. Most of these studies vary in their dosing regimens and infusion protocols. Some of the earlier trials utilized prolonged anesthetic dosages to induce a "ketamine coma" [15]. In an open label, prospective study by Kiefer et al. (2008), refractory CRPS patients were anesthetized with ketamine in the intensive care unit for 5 straight days [15]. At 1 month post-infusion, all subjects showed a significant reduction in self-reported pain. At 6 months post-infusion, 16 of 20 subjects had complete remission from CRPS while the 4 patients who relapsed at 6 months still reported significant pain relief. The authors concluded that anesthetic doses of ketamine can provide prolonged pain relief in refractory CRPS patients and that a randomized controlled trial is warranted to confirm its efficacy [15].

However, the intensive nature of the intervention employed by this study raised concerns over adverse events and safety. Seventeen of 20 patients required intubation and mechanical ventilation over the duration of their infusions. Post-infusion, all subjects experienced psychotomimetic side effects of ketamine including anxiety, dysphoria, nightmares, and insomnia. Most side effects resolved after 1 week post-treatment, however they persisted for over 1 month in 5 patients. In addition, 14 of the 20 patients experienced infectious complications (respiratory and urinary) during their intensive care unit stay. Some patients also experienced significant weight loss, as well as temporary muscle weakness and ataxia following treatment $[14,15]$.

Due to concerns over morbidity and mortality in intensive care medicine as well as the high cost, the intervention used by Kiefer et al. (2008) has been largely regarded as infeasible [25]. Very few centres or clinicians around the world would offer the "ketamine coma" as a reasonable approach to managing CRPS [26].

Subsequent trials have shifted their focus to potentially safer protocols for ketamine infusions by utilizing prolonged, but subanesthetic dosages that do not require intensive monitoring. To date, two double-blind, randomized controlled trials have examined the efficacy of IV subanesthetic ketamine in CRPS $[16,17]$. Sigter- 
mans et al. (2009) used a continuous, 4.2-day inpatient protocol on 60 CRPS patients [16]. Thirty patients received subanesthetic ketamine, which was titrated to analgesia and kept to a maximum of $0.43 \mathrm{mg} / \mathrm{kg} / \mathrm{h}$, while the placebo group was given normal saline. Results indicated a significant decrease in self-reported pain in the ketamine group compared to placebo, for up to 11 weeks post-infusion. Functional outcomes were assessed using the Radboud Skills Questionnaire (a quantitative measure of ability to perform activities of daily living with the affected limb) and the Walking Ability Questionnaire (evaluation of the impact of pain on walking performance). Interestingly, patients receiving ketamine did not report a significant increase in any of these functional outcomes compared to placebo, even in the weeks following treatment. The authors suggested that in addition to the pain relief achieved with ketamine, adjunctive physical rehabilitation may be necessary to regain functional ability [16].

Despite promising results, this study was not without criticism. Not surprisingly, there was a high incidence of side effects in the treatment group. For instance, $93 \%$ of patients receiving ketamine experienced some form of psychotomimetic effect, $63 \%$ experienced nausea, and $47 \%$ experienced vomiting [16]. The authors state that most patients found the intensity of the side effects acceptable. However, it is important to note that there were also 2 subjects who withdrew from the study because of intolerable psychotomimetic effects [16].

Another point of criticism was the use of a weak placebo (normal saline), which may have compromised blinding in terms of treatment allocation. Both the participants and investigators became aware of the treatment assignments based on the presence or absence of psychotomimetic side effects. In fact $74 \%$ of patients and $88 \%$ of investigators correctly guessed the administered treatment when surveyed after the trial [16]. Despite these weak points, this infusion protocol represents a more feasible alternative to the ketamine coma technique described earlier [16].

In another double-blind, placebo-controlled trial by Schwartzman et al. (2009), the infusion protocol was made suitable for outpatient therapy [17]. Nineteen CRPS patients who failed conventional therapies were recruited and infused intravenously with either ketamine or normal saline (placebo) 4 hours daily for 10 days ( 5 straight days, followed by a 2 day weekend break, and 5 straight days again). Ketamine was titrated up to a maximum of $0.35 \mathrm{mg} / \mathrm{kg} / \mathrm{h}$. In addition, all patients, including those in the placebo group, received midazolam, a short-acting benzodiazepine, and clonidine, a centrally-acting sympatholytic agent. These agents were included for 2 reasons: 1) midazolam and clonidine may help control psychotomimetic side effects; and 2) given that the study patients were ketamine naive, these agents may serve as an active placebo by producing noticeable side effects and thus resulting in a more robust blinding process [27].
Pain was assessed before and after the infusion protocol using a numeric rating scale $(0-10)$ that assessed various pain parameters including: 1) pain in response to deep pressure, 2) joint pain, 3 ) degree to which pain interferes with general activity, 4) pain in the most affected area, 5) burning pain, 6) pain in response to light touch, and 7) overall pain. In the ketamine group, a significant score improvement was found in the latter four parameters. The greatest improvement was noted during the first month post-treatment. On average, pain scores decreased by 27\% 1-4 weeks post-treatment, whereas the placebo group showed no significant improvement in any of the pain parameters. Similar results were demonstrated using the short-form McGill pain questionnaire. The average decrease in McGill pain score was $35 \%$, which lasted 12 weeks post-treatment [17].

A closer look at the parameters of the pain questionnaire shows that no significant improvement was found for the "degree to which pain interferes with general activity". General activity levels of patients, which were recorded pre- and post-treatment by accelerometer-equipped wristwatches, showed no significant differences. Subjects also reported no change in quality of life, as measured by the American Chronic Pain Association quality of life questionnaire. Overall, this suggests that there was no improvement in function or return to activity following the infusions. Therefore, similar to the study by Sigtermans et al. (2009), decreased pain levels were not accompanied by improvements in functional outcomes $[16,17]$.

Although the use of midazolam and clonidine as an active placebo presumably strengthened the blinding process of this study, no post-treatment data was reported on how well subjects were able to guess their assignment. The use of midazolam and clonidine did, however, help address the issue of psychotomimetic side effects. No participant from the ketamine group reported psychotomimetic side effects. The authors concluded that the concomitant use of midazolam and clonidine may effectively minimize the undesirable side effects of ketamine [17].

Compared to Sigtermans et al. (2009), this study by Schwartzman et al. (2009) appears to adopt a safer infusion protocol and a stronger experimental method [16,17]. However, there is one major criticism of this research trial that warrants discussion. Power analysis originally indicated 20 subjects per treatment arm; however the trial was terminated early, leaving a small sample size of only 19 subjects (9 ketamine, 10 placebo). The authors claimed that over the 2-year course of the trial, their continued experience with outpatient ketamine treatments indicated that higher doses (up to twice the maximum limit set by the trial) could be used to achieve much greater and longer lasting pain relief without added complications [17]. Therefore, the "principle of beneficence in clinical research" was cited to justify the early termination of the trial [27]. Although many of the pain 
parameters showed statistically significant improvements in the study by Schwartzman et al. (2009), it is well known that small sample sizes are susceptible to overestimating treatment effects $[17,28,29]$. Therefore, a larger trial with sufficient power may be necessary to confirm the true efficacy of this infusion protocol.

In summary, the studies discussed above illustrate the different approaches to ketamine infusions to treat CRPS. The analgesic effects of ketamine are dose-dependent and the ketamine coma may offer the longest duration of pain relief. However, infusion with subanesthetic doses of ketamine is more feasible, and the outpatient protocol by Schwartzman et al. (2009) may be the most practical and cost-effective to implement [17]. Treatment with this protocol appears to provide pain relief that may last up to 12 weeks post-infusion. It is unknown whether long-term remission is possible, but repeated infusions would likely be necessary to maintain analgesia. The two randomized controlled trials discussed represent the best available evidence thus far $[16,17]$. However, both studies contain their own respective weaknesses. Despite promising results, the evidence across all studies is not strong and there is a need for larger, long-term studies to confirm the efficacy, as well as the long-term safety of ketamine infusions.

\section{LONG-TERM SAFETY}

There is a growing number of case reports related to the potential toxicity of ketamine [30-33]. Unfortunately, long-term safety data on the use of ketamine in chronic pain does not exist. Much of our current understanding on long-term ketamine toxicity comes from retrospective studies of chronic ketamine abusers $[21,34]$. Given that patients with refractory CRPS may be subject to multiple ketamine infusions over time, the toxicity associated with chronic ketamine abuse may provide pertinent information [34].

\section{Neurotoxicity}

Experimental models involving rodents and rhesus monkeys have demonstrated neurodegenerative effects after 24 hours of continuous ketamine administration $[35,36]$. It is unknown whether these effects are reversible. Although observations from animal studies have not been confirmed in humans, concerns remain on whether ketamine-associated neurotoxicity may cause potential deficits in neurocognitive processes.

Several studies have shown that long-term ketamine abuse is associated with deficits in cognitive function, especially in the form of impaired memory and attention [37,38]. One longitudinal study involving long-term ketamine abusers showed that psychotomimetic symptoms could persist even after complete cessation of the drug for 3 years [39]. Taken together, these suggest that permanent damage to the brain may be possible. An MRI study by Liao et al. (2011) observed significant decreases in gray matter volume of the bilateral frontal cortex in chronic ketamine abusers compared to healthy volunteers [40]. This suggests a structural basis for the observed deficits in cognitive function. However, these findings must be interpreted cautiously, since the study was retrospective and therefore unable to establish causation between ketamine and the observed structural changes.

\section{Urological toxicity}

Another harm which has been documented in chronic ketamine abusers is urological dysfunction, including ulcerative cystitis [41]. One case series described severe genitourinary symptoms including urgency, frequency, hematuria, and post-mictural pain in daily ketamine abusers [42]. Subsequent case reports in the literature have described similar incidences of urological toxicity in patients receiving ketamine in the clinical setting. For instance, Gregoire et al. (2008) reported a 16-year-old CRPS patient who presented with cystitis following the use of oral ketamine $(8 \mathrm{mg} /$ $\mathrm{kg} /$ day) as an analgesic adjunct after just 9 days of treatment [30]. Storr et al. (2009) described 3 cases of palliative pain patients who developed significant urological symptoms following administration of oral ketamine for 5-12 months [31].

The exact mechanism by which ketamine-induced cystitis occurs is unknown. It was once thought that all urological symptoms would resolve upon discontinuation of chronic ketamine use. However, a recent longitudinal study involving 44 ketamine abusers found that in $90 \%$ of subjects, urological symptoms persisted for 8 months following ketamine cessation [43].

\section{Hepatotoxicity}

There are also reports of potential liver toxicity associated with prolonged ketamine use. In a trial by Noppers et al. (2011), 3 out of 6 CRPS patients who were scheduled to receive 2 continuous 100-hour infusions (16 days apart) of subanesthetic ketamine developed signs of liver injury [32]. During the second infusion, all 3 patients had liver enzyme levels that were 3 times the upper limit of normal. The infusions were discontinued and the patients' enzyme levels slowly returned to normal within 3 months. However, concerns over hepatotoxicity were significant enough to warrant early termination of this trial.

Other studies using either anesthetic or subanesthetic doses of ketamine have reported mixed observations. For example, Kiefer et al. (2008) reported similar elevations in liver enzymes in 16 out of 20 subjects after the 5-day "ketamine coma" protocol, whereas Sigtermans et al. (2009) reported no elevations in liver enzymes in any of the 30 subjects who received a continuous, 4.2-day subanesthetic ketamine infusion $[15,16]$. Schwartzman et al. (2009) either did not measure liver enzymes or neglected 
to report them in their study [17].

The mechanism of liver injury is unclear, but the risk may be higher with repeated infusions [34]. Currently, there are no long-term follow-up studies documenting hepatotoxicity from multiple ketamine infusions. However, a recent report described a case of liver cirrhosis that was potentially attributed to an 8-year history of oral ketamine use for managing chronic facial pain [33]. Unfortunately, the validity of this claim remains unclear and further research is needed.

\section{CONCLUSIONS}

CRPS is a potentially debilitating condition with limited therapeutic options. Therefore, any treatment with the promise of pain relief warrants serious consideration. Ketamine infusions may be an effective option for providing analgesia in patients with refractory CRPS. Unfortunately, the current evidence for this is not strong, and there is no consensus on the most effective infusion protocol. However, the outpatient subanesthetic regimen used by Schwartzman et al. (2009) likely demonstrates the best balance of efficacy and practicality based on the available data [17].

Importantly, both physicians and patients must be aware that there are potential long-term safety risks associated with ketamine infusions and further research is needed to ascertain the implications of prolonged ketamine treatment. In the meantime, ketamine infusions should be reserved for refractory cases of CRPS and only after careful consideration of both the risks and benefits.

For patients who do undergo ketamine treatment, proper monitoring must be ensured. Given the psychotomimetic properties of ketamine, a psychological evaluation prior to therapy may be prudent. Careful monitoring for urological symptoms and signs of hepatotoxicity is also advisable, especially for patients exposed to multiple treatments.

Finally, studies have consistently shown no measureable increases in functional outcomes following treatment with ketamine, despite reductions in pain scores $[16,17]$. This suggests that pain relief may be just one component in the overall complex management of CRPS. In addition to adequate pain control, a functional rehabilitation program may be necessary to help restore quality of life in patients with refractory CRPS.

\section{REFERENCES}

1. Schasfoort FC, Bussmann JB, Stam HJ. Impairments and activity limitations in subjects with chronic upper-limb complex regional pain syndrome type I. Arch Phys Med Rehabil. 2004;85(4):557-566.

2. Freedman M, Greis AC, Marino L, Sinha AN, Henstenburg J. Complex regional pain syndrome: diagnosis and treatment. Phys Med Rehabil Clin $\mathrm{N}$ Am. 2014;25(2):291-303.

3. Harden RN, Bruehl S, Stanton-Hicks M, Wilson PR. Proposed new diagnostic criteria for complex regional pain syndrome. Pain Med. 2007;8(4):326-331.

4. Sandroni P, Benrud-Larson LM, McClelland RL, Low PA. Complex regional pain syndrome type I: incidence and prevalence in Olmsted county, a population-based study. Pain. 2003;103(1-2):199-207.

5. Wasner G, Schattschneider J, Binder A, Baron R. Complex regional pain syndrome--diagnostic, mechanisms, CNS involvement and therapy. Spinal Cord. 2003;41(2):61-75.

6. Schwartzman RJ, Erwin KL, Alexander GM. The natural history of complex regional pain syndrome. Clin J Pain. 2009;25(4):273-280.

7. De Mos M, de Bruijn AG, Huygen FJPM, Dieleman JP, Stricker BHC, Sturkenboom MCJM. The incidence of complex regional pain syndrome: a population-based study. Pain. 2007;129(1-2):12-20.

8. Naleschinski D, Baron R. Complex regional pain syndrome type I: neuropathic or not? Curr Pain Headache Rep. 2010;14(3):196-202.

9. Bruehl S. An update on the pathophysiology of complex regional pain syndrome. Anesthesiology. 2010;113(3):713-725.

10. Tran DQH, Duong S, Bertini P, Finlayson RJ. Treatment of complex regional pain syndrome: a review of the evidence. Can J Anaesth. 2010;57(2):149166.

11. Cossins L, Okell RW, Cameron H, Simpson B, Poole HM, Goebel A. Treatment of complex regional pain syndrome in adults: a systematic review of randomized controlled trials published from June 2000 to February 2012. Eur J Pain. 2013;17(2):158-173.

12. Forouzanfar T, Köke AJ, van Kleef M, Weber WEJ. Treatment of complex regional pain syndrome type I. Eur J Pain. 2002;6(2):105-122.

13. Fisher K, Coderre TJ, Hagen NA. Targeting the N-methyl-D-aspartate receptor for chronic pain management. Preclinical animal studies, recent clinical experience and future research directions. J Pain Symptom Manage. 2000;20(5):358-373.

14. Schwartzman RJ, Alexander GM, Grothusen JR. The use of ketamine in complex regional pain syndrome: possible mechanisms. Expert Rev Neurother. 2011;11(5):719-734.

15. Kiefer R-T, Rohr P, Ploppa A, et al. Efficacy of ketamine in anesthetic dosage for the treatment of refractory complex regional pain syndrome: an openlabel phase II study. Pain Med. 2008;9(8):1173-1201.

16. Sigtermans MJ, van Hilten JJ, Bauer MCR, et al. Ketamine produces effective and long-term pain relief in patients with Complex Regional Pain Syndrome Type 1. Pain. 2009;145(3):304-311.

17. Schwartzman RJ, Alexander GM, Grothusen JR, Paylor T, Reichenberger E, Perreault M. Outpatient intravenous ketamine for the treatment of complex regional pain syndrome: a double-blind placebo controlled study. Pain. 2009;147(1-3):107-115.

18. Shirani P, Salamone AR, Schulz PE, Edmondson E. Ketamine treatment for intractable pain in a patient with severe refractory complex regional pain syndrome: a case report. Pain Physician. 2008;11(3):339-342.

19. Goebel A, Turner-Strokes L. Complex regional pain syndrome in adults: UK guidelines for diagnosis, referral and management in primary and secondary care. London: Royal College of Physicians; 2012. $60 \mathrm{p}$.

20. Domino EF. Taming the ketamine tiger. 1965. Anesthesiology. 2010;113(3):678-684.

21. Morgan CJ, Curran HV. Ketamine use: a review. Addiction. 2012;107(1):2738.

22. Grant IS, Nimmo WS, Clements JA. Pharmacokinetics and analgesic effects of i.m. and oral ketamine. Br J Anaesth. 1981;53(8):805-810.

23. Persson J. Wherefore ketamine? Curr Opin Anaesthesiol. 2010;23(4):455460.

24. McCartney CJL, Sinha A, Katz J. A qualitative systematic review of the role of $\mathrm{N}$-methyl-D-aspartate receptor antagonists in preventive analgesia. Anesth Analg. 2004;98(5):1385-1400.

25. Koffler SP, Hampstead BM, Irani F, et al. The neurocognitive effects of 5 day anesthetic ketamine for the treatment of refractory complex regional pain syndrome. Arch Clin Neuropsychol. 2007;22(6):719-729.

26. Pickering AE, McCabe CS. Prolonged ketamine infusion as a therapy for complex regional pain syndrome: synergism with antagonism? Br J Clin Pharmacol. $2014 ; 77(2): 233-238$.

27. Schwartzman RJ. Response to Drs. Bell and Moore commentary regarding the use of intravenous ketamine for CRPS. Pain. 2010;151(2):554-555; author reply 556-557.

28. Bell RF, Moore R. Intravenous ketamine for CRPS: Making too much of too little? Pain. 2010;150(1):10-11.

29. Button KS, loannidis JPA, Mokrysz C, et al. Power failure: why small sample size undermines the reliability of neuroscience. Nat Rev Neurosci. 2013;14(5):365-376.

30. Gregoire M. A pediatric case of ketamine-associated cystitis (Letter-to-the- 
Editor RE: Shahani R, Streutker C, Dickson B, et al: Ketamine-associated ulcerative cystitis: a new clinical entity. Urology 69: 810-812, 2007). Urology. 2008;71(6):1232-1233.

31. Storr TM, Quibell R. Can ketamine prescribed for pain cause damage to the urinary tract? Palliat Med. 2009;23(7):670-672.

32. Noppers IM, Niesters M, Aarts LPHJ, et al. Drug-induced liver injury following a repeated course of ketamine treatment for chronic pain in CRPS type 1 patients: a report of 3 cases. Pain. 2011;152(9):2173-2178.

33. Bevan R, Burke D. Ketamine as a possible cause of cirrhosis in a patient with chronic pain. Frontline Gastroenterol. 2013;5:208-210.

34. Bell RF. Ketamine for chronic noncancer pain: concerns regarding toxicity. Curr Opin Support Palliat Care. 2012;6(2):183-187.

35. Jevtovic-Todorovic V, Carter LB. The anesthetics nitrous oxide and ketamine are more neurotoxic to old than to young rat brain. Neurobiol Aging. 2005;26(6):947-956.

36. Slikker W, Zou X, Hotchkiss CE, et al. Ketamine-induced neuronal cell death in the perinatal rhesus monkey. Toxicol Sci. 2007;98(1):145-158. .

37. Morgan CJ, Curran HV. Acute and chronic effects of ketamine upon human memory: a review. Psychopharmacology (Berl). 2006;188(4):408-424.

38. Morgan CJ, Muetzelfeldt L, Curran HV. Consequences of chronic ketamine self-administration upon neurocognitive function and psychological wellbeing: a 1-year longitudinal study. Addiction. 2010;105(1):121-133.

39. Morgan CJ, Monaghan L, Curran HV. Beyond the K-hole: a 3-year longitudinal investigation of the cognitive and subjective effects of ketamine in recreational users who have substantially reduced their use of the drug. Addiction. 2004;99(11):1450-1461.

40. Liao Y, Tang J, Corlett PR, et al. Reduced dorsal prefrontal gray matter after chronic ketamine use. Biol Psychiatry. 2011;69(1):42-48.

41. Chiew YW, Yang CS. Disabling frequent urination in a young adult. Ketamineassociated ulcerative cystitis. Kidney Int. 2009;76(1):123-124.

42. Shahani R, Streutker C, Dickson B, Stewart RJ. Ketamine-associated ulcerative cystitis: a new clinical entity. Urology. 2007;69(5):810-812.

43. Cheung RYK, Chan SSC, Lee JHS, Pang WL, Choy KW, Chung TKH. Urinary symptoms and impaired quality of life in female ketamine users: persistence after cessation of use. Hong Kong Med J. 2011;17(4):267-273. 